\title{
Aggregatibacter actinomycetemcomitans
}

National Cancer Institute

\section{Source}

National Cancer Institute. Aggregatibacter actinomycetemcomitans. NCI Thesaurus.

Code C86133.

A species of facultatively anaerobic, Gram negative, coccobacilli shaped bacteria assigned to the phylum Proteobacteria. This bacteria is non motile, catalase and oxidase positive, does not hydrolyze ortho nitrophenyl beta galactosidase, urease negative and acid is produced from glucose, maltose, mannose and fructose. A. actinomycetemcomitans is an oral commensal in humans and Old World primates, and may be a cause of periodontal disease. 